\title{
Impact of COVID-19 on U.S. and Canadian neurologists' therapeutic approach to multiple sclerosis: a survey of knowledge, attitudes, and practices
}

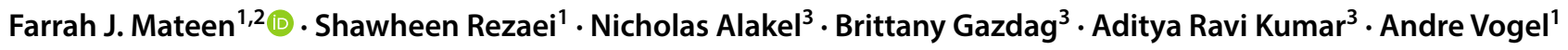

Received: 26 May 2020 / Revised: 27 June 2020 / Accepted: 1 July 2020 / Published online: 7 July 2020

(c) Springer-Verlag GmbH Germany, part of Springer Nature 2020

\begin{abstract}
Objective To report the understanding and decision-making of neuroimmunologists and their treatment of patients with multiple sclerosis (MS) during the early stages of the SARS-CoV-2 (COVID-19) outbreak.

Methods A survey instrument was designed and distributed online to neurologists in April 2020.

Results There were 250 respondents (response rate 21.8\%). 243 saw $>=10$ MS patients in the prior 6 months (average 197 patients) and were analyzed further (92\% USA, 8\% Canada; average practice duration 16 years; 5\% rural, 17\% small city, $38 \%$ large city, $40 \%$ highly urbanized). Patient volume dropped an average of $79 \%$ (53-11 per month). 23\% were aware of patients self-discontinuing a DMT due to fear of COVID-19 with $43 \%$ estimated to be doing so against medical advice. $65 \%$ of respondents reported deferring $>=1$ doses of a DMT (49\%), changing the dosing interval (34\%), changing to home infusions (20\%), switching a DMT (9\%), and discontinuing DMTs altogether (8\%) as a result of COVID-19. Changes in DMTs were most common with the high-efficacy therapies alemtuzumab, cladribine, ocrelizumab, rituximab, and natalizumab. 35\% made no changes to DMT prescribing. 98\% expressed worry about their patients contracting COVID-19 and 78\% expressed the same degree of worry about themselves. $>50 \%$ believed high-efficacy DMTs prolong viral shedding of SARS-CoV-2 and that B-cell therapies might prevent protective vaccine effects. Accelerated pace of telemedicine and practice model changes were identified as major shifts in practice.

Conclusions Reported prescribing changes and practice disruptions due to COVID-19 may be temporary but could have a lasting influence on MS care.
\end{abstract}

Keywords Multiple sclerosis · COVID-19 $\cdot$ Immunosuppression $\cdot$ Health behaviors $\cdot$ Neuroimmunology $\cdot$ Neurologist . Disease-modifying therapy

\section{Introduction}

There are $>725,000$ people living with multiple sclerosis (MS) in the United States of America and approximately 93,000 in Canada, with the majority treated with

Electronic supplementary material The online version of this article (https://doi.org/10.1007/s00415-020-10045-9) contains supplementary material, which is available to authorized users.

Farrah J. Mateen

fmateen@mgh.harvard.edu

1 Department of Neurology, Massachusetts General Hospital, 165 Cambridge Street, Office 627, Boston, MA 02114, USA

2 Harvard Medical School, Boston, USA

3 ZoomRx, Boston, USA disease-modifying therapies (DMTs) [1,2]. The hallmark of treatment in MS is immunosuppression, or in some cases immunomodulation, which has a very strong evidence base but must be continuously evaluated for its risk-benefit ratio in patients across the disease course and throughout the lifespan. There are now 20 FDA-approved DMTs for multiple sclerosis with several additional therapies used in practice and others in the therapeutic pipeline [3]. In general, people with MS have a higher risk than the general population for multiple infectious diseases, particularly when treated with high-efficacy DMTs [4, 5].

In March 2020, the first case of the new SARSCoV-2-COVID-19 virus was reported in the USA [6]; however, earlier cases in the USA, prior to widespread recognition, are now confirmed [7]. By the end of April 2020, there were more than $>1.5$ million confirmed cases in the 
USA and >90,000 COVID-19-attributed deaths [8]. At the time of this report, the USA has more cases of COVID-19 than any other country in the world and the highest absolute number of deaths. This situation, previously unimaginable only a few months earlier, has profoundly influenced inter alia neurological care $[9,10]$.

Immunosuppression is a reported risk factor for developing COVID-19 by the U.S. Centers for Disease Control and Prevention [11]. Immunosuppression or immunomodulation could impact patient prognosis if coronavirus infection occurs. However, the cases reported among people living with MS are still being gathered and understood, with only anecdotal reports available presently. Some information can be drawn through informal channels including social media. Larger efforts are ongoing, but a synthesized report on MS patients and their outcomes was not yet published when our study commenced. General guidelines are emerging [12,13], but were generally limited at the time of our study. Amidst this pandemic and declared national emergency in the USA, with a parallel response in Canada, neuroimmunologists are making important decisions and adjudicating complex situations of risk for their patients living with MS.

This study reports the knowledge, attitudes, and practices of neurologists treating MS. We attempt to synthesize the deductions and strategies of a large sample of subspecialized neurologists in United States and Canada at the early height of the pandemic, surveyed online beginning in mid-April 2020. Where discrepancies exist, we attempt to show the range of responses on a particular issue. The overall objectives of this study were threefold: (1) to report the range of impacts of COVID-19 on neuroimmunologists' practice across the USA and Canada; (2) to probe the MS DMT prescribing decisions and planning of neuroimmunologists in the setting of a viral pandemic; and (3) determine the unmet needs and sources of uncertainty that dominate the care of MS patients. Understanding of the present situation could identify points of contention among experts and reveal shared uncertainties that can be addressed through targeted research. Consensus on current best practices among neurologists could further support patients, prescribers, and practices in future public health emergencies.

\section{Methods}

\section{Ethics approvals}

The study received expedited review and approval by the Massachusetts General Hospital's Institutional Review Board.

\section{Survey design}

A new survey instrument was designed by the authors to query the most urgent and prevalent issues that MSfocused neurologists were perceived to experience in the first approximately 6 weeks of the recognized COVID-19 outbreak in North America. The survey questions were based on evolving reports, clinical anecdotes and experiences, and incorporation of patient-based queries until early April 2020, when the final version of the survey instrument was confirmed and IRB approved.

The thematic focus of the questions was on the knowledge, attitudes, and practices of MS DMT prescribers. Rather than emphasizing fact checking, the survey queried awareness of local COVID-19 cases and patients' health practices, impressions and worries on the risk of COVID19 to patients taking MS DMTs, and prescribing patterns in various special situations, naming the exact DMTs. As an example, issues related to older patients with MS were queried, defined as age 55 years and older (given the usual age cutoff for most DMT trials to date) or 60 years and older (given the Centers for Disease Control and Prevention's general consideration of people aged 60 years and older as a higher risk group) [11], depending on the question. The survey instrument is available as Appendix 1.

\section{Survey implementation}

Eligibility for the survey was determined based on screening questions. Eligible respondents must have seen a critical number of MS patients (pre-defined as a minimum of $10 \mathrm{MS}$ patients per month in the past 6 months). Only physicians who self-declared a subspecialty expertise in MS were eligible. The survey included a combination of fillin-the-blank, multiple choice, and open-ended questions. Pilot testing was performed by the authors in a sample of 12 participants, and the accuracy and completeness of the responses were reviewed by the authors prior to wider distribution.

\section{Survey distribution}

Surveys were distributed through an email link, in English, to a known panel of MS prescribers who have answered previous surveys online as well as to publicly available e-mail addresses of neuroimmunology-focused U.S. and Canadian neurologists, from April 14, 2020 to May 3, 2020, an approximately 3-week period. A single reminder was sent. A sample size of 250 U.S. and Canadian respondents was targeted or a study end date of May 
Table 1 Demographic information of neurologists $(n=243)$

\begin{tabular}{lrc}
\hline Demographic information & Frequency (\# providers) & Percentage (\%) \\
\hline Country of residence & 224 & 91.8 \\
USA & 19 & 7.8 \\
Canada & & \\
Age distribution & 22 & 9.1 \\
25-35 years old & 80 & 32.9 \\
35-45 years old & 61 & 25.1 \\
45-55 years old & 63 & 25.9 \\
55-65 years old & 17 & 7.0 \\
Greater than 65 years old & & 4.9 \\
Practice location & 12 & 17.2 \\
Rural area (less than 50,000 residents) & 42 & 38.1 \\
Smaller city (50,000 to 100,000 residents) & 93 & 39.3 \\
Larger city (100,000 to 1,000,000 residents) & 96 & \\
Highly urban area (> 1,000,000 residents) & & 41.4 \\
Practice setting & 101 & 5.7 \\
Academic Hospital & 14 & 20.5 \\
Community Hospital & 50 & 23.8 \\
Multi-specialty group or partnership & 58 & 8.2 \\
Single specialty group or partnership & 20 & \\
Solo private practice & & \\
\hline
\end{tabular}

5, whichever came first. Invitation posts to public forums were made including the Canadian Network of MS Clinics and the American Academy of Neurology's MS, NeuroInfectious Disease, and Solo and Small Practices Section.

All responses were collected anonymously with no identifiable information gathered on the respondents by the study team. The questions were entered electronically into the ZoomRx platform. Each page required complete responses before advancing to the next page. Where questions were relevant to only a specific subgroup of respondents who replied "yes" or "no" to prior questions, subsamples of the full sample size are reported. Respondents who completed the survey in full were each offered an honorarium of 50 USD.

\section{Data analysis}

Quality control of the survey responses was performed. Responses were analyzed by each question and summarized by their proportions (percentages) for categorical variables and the mean, median, and percentiles for continuous variables. Free text responses were reviewed by the authors and reported qualitatively.

\section{Results}

\section{Response rate and respondents}

There were 250 responses (estimated response rate 21.8\%) who cared for MS patients in the past 6 months. Characteristics of the respondents including the practice setting are provided in Table 1. Respondents came from 48 of the 50 U.S. states and Canada. U.S-based respondents came from many of the states with the highest number of incident COVID-19 cases, recognized through virus testing: New York (8\%), California (8\%), Massachusetts (8\%), Florida (7\%), and Pennsylvania (5\%). The average number of years in practice was 16 .

\section{Practice changes}

There were 243 respondents who had seen at least 10 MS patients in the prior 6 months and analyzed further. The average number of MS patients seen by the 


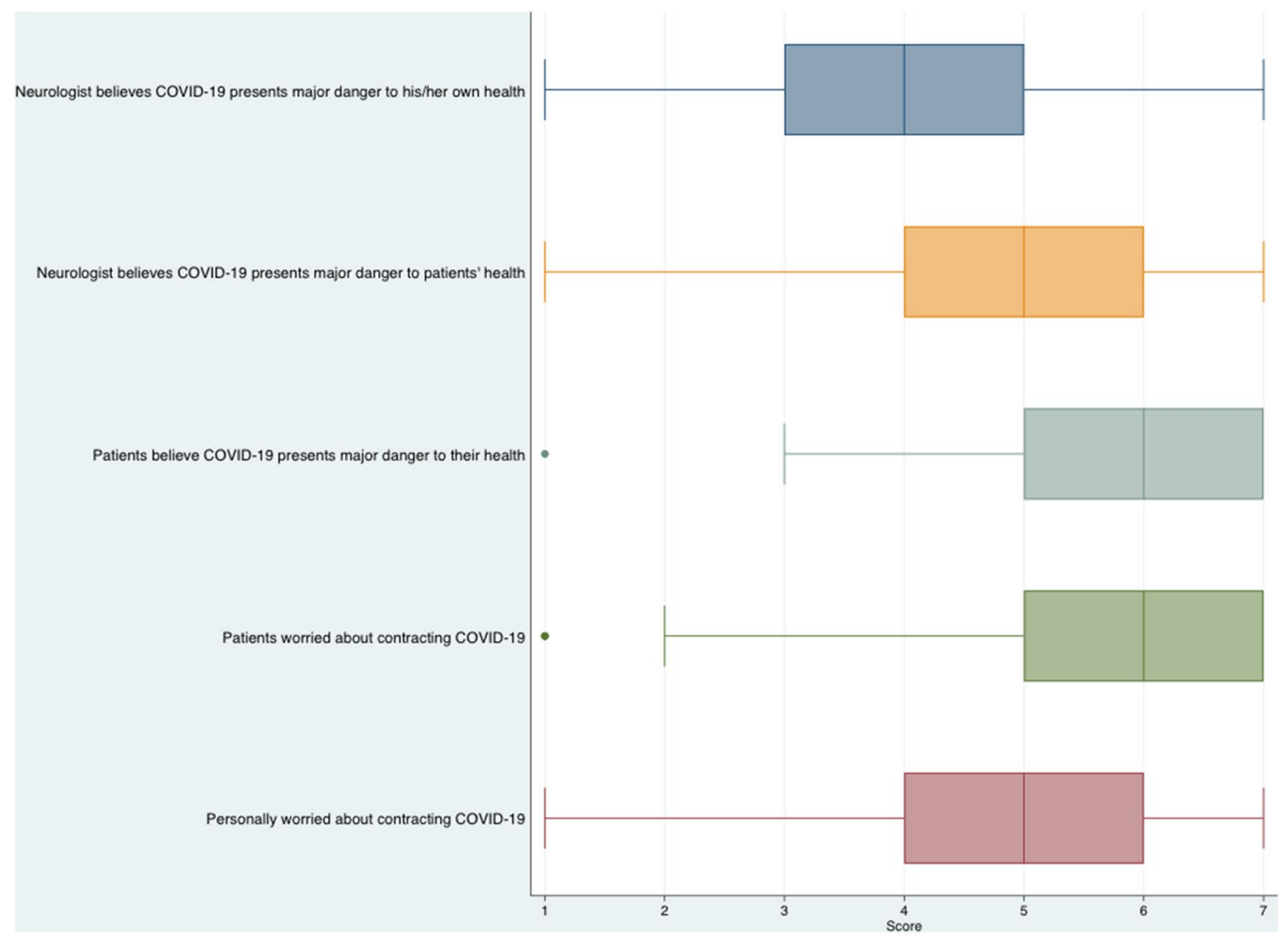

Fig. 1 Neurologists' $(n=243)$ rating of personal and patient attitudes toward COVID-19

respondents was 197 in the prior 6 months with 53 in a "typical month" but 11 in the last month (in roughly the month of April 2020). This reflected an average decrease in 42 MS patients (79\% drop) seen by neurologists during the COVID-19 epidemic peak. $97 \%$ of respondents were aware of a case of COVID-19 in their community, town, or city.

The degree of worry for MS (and neuromyelitis optica) patients and themselves as practitioners becoming infected by the novel coronavirus is given in Fig. 1. Most respondents (98\%) expressed an average or more worry about their patients contracting SARS-CoV-2 and 78\% expressed the same degree about themselves getting the virus with $90 \%$ considering it a danger to their patients' health and $74 \%$ to their own health. Moreover, $15 \%$ of neurologists believed that COVID-19 is a major danger to their MS (or neuromyelitis optica) patients' health with $11 \%$ felt the same for their own health. $93 \%$ of neurologists had conducted a televisit (telephone and/or video) directly as a result of the COVID-19 epidemic, with an estimated $82 \%$ of all visits already being conducted via telemedicine by the time of the survey.

\section{Patient-initiated changes}

Physicians estimated that $86 \%$ of patients on average made changes to their daily lives and health behaviors due to SARS-CoV-2 virus with the most common decision being "social distancing" (98\%) followed by avoiding travel (94\%), not going to work (89\%), wearing a medical mask (89\%), social isolation (83\%), self-quarantine (71\%), and wearing gloves (58\%).

Among the $23 \%$ of specialists stating that they are aware of any of their MS patients self-discontinuing DMTs due to worry about contracting the virus, an estimated $7 \%$ of patients had self-discontinued their prescribed DMT in the setting of COVID-19. In $43 \%$ of cases, this was against medical advice, $28 \%$ it was upon medical advice, and $28 \%$ it was neutral (neither against advice or with advice to stop).

\section{Prescribing choices}

Nearly all physician respondents (94\%) believe that their MS patients are at an increased risk of acquiring COVID-19 compared to the general population, with $41 \%$ considering 
Table 2 Neurologist prescribing patterns pre- and post-COVID-19

\begin{tabular}{|c|c|c|c|c|}
\hline Type of treatment & $\begin{array}{l}\text { MS patients receiving } \\
\text { treatment pre-COVID-19 } \\
(\text { mean \%) } \\
(n=243)\end{array}$ & $\begin{array}{l}\text { Not consider prescribing to } \\
\text { a newly diagnosed patient } \\
\text { or switching patient in light } \\
\text { of COVID-19 }(\%) \\
(n=243)\end{array}$ & $\begin{array}{l}\text { Consider starting MS } \\
\text { patients on in light of } \\
\text { COVID-19 }(\%) \\
(n=243)\end{array}$ & $\begin{array}{l}\text { Perceive as safer to use } \\
\text { in light of COVID-19 } \\
(\%) \\
(n=132)\end{array}$ \\
\hline Aubagio (teriflunomide) & 9 & 5 & 52 & 50 \\
\hline Chronic steroids & 2 & 31 & 3 & 1 \\
\hline $\begin{array}{l}\text { Copaxone or glatopa (glati- } \\
\text { ramer acetate) }\end{array}$ & 14 & 4 & 77 & 95 \\
\hline Gilenya (fingolimod) & 11 & 18 & 36 & 8 \\
\hline Interferons & 13 & 6 & 63 & 80 \\
\hline Lemtrada (alemtuzumab) & 1 & 61 & 5 & N/A \\
\hline Mavenclad (cladribine) & 2 & 47 & 8 & 1 \\
\hline Mayzent (siponimod) & 2 & 18 & 24 & 5 \\
\hline Ocrevus (ocrelizumab) & 17 & 38 & 31 & 3 \\
\hline Ofatumumab & N/A & 20 & 11 & 2 \\
\hline $\begin{array}{l}\text { Recurrent intravenous } \\
\text { immunoglobulin (IVIg) }\end{array}$ & 1 & 8 & 15 & 17 \\
\hline $\begin{array}{l}\text { Recurrent plasma exchange } \\
\text { (PLEX) }\end{array}$ & 1 & 10 & 8 & 8 \\
\hline Rituxan (rituximab) & 3 & 35 & 16 & 4 \\
\hline $\begin{array}{l}\text { Tecfidera (dimethyl fuma- } \\
\text { rate) }\end{array}$ & 17 & 9 & 58 & 31 \\
\hline Tysabri (natalizumab) & 8 & 34 & 36 & 26 \\
\hline $\begin{array}{l}\text { Vumerity (diroximel fuma- } \\
\text { rate) }\end{array}$ & 1 & 6 & 32 & 18 \\
\hline Zeposia (ozanimod) & 0 & 14 & 16 & 3 \\
\hline No treatment & 6 & N/A & N/A & N/A \\
\hline None of the above & 4 & 17 & 4 & N/A \\
\hline
\end{tabular}

this risk slightly increased, $43 \%$ considering it moderately increased, and $10 \%$ considering it significantly increased.

Prior to COVID-19, respondents estimated that their MS patients were on average taking injectable agents $(25 \%)$, oral therapies (38\%), and infused therapies (26\%) (Table 2). An estimated $6 \%$ of MS patients were untreated with any DMT. $5 \%$ of MS patients were on more than one immunosuppressive agent. $4 \%$ of all MS patients were estimated to be presently enrolled in a clinical trial. Specific DMT prescribing patterns before and after COVID-19 are given in Table 2. In general, $54 \%$ of respondents believed that certain DMTs are safer during the COVID-19 pandemic than others; $4 \%$ did not think so; and $42 \%$ were unsure. The DMTs believed to be safer during the pandemic were glatiramer acetate $(95 \%)$, interferons $(80 \%)$, and teriflunomide $(50 \%)$. DMTs that neurologists would consider starting and not consider starting in newly diagnosed MS patients are given in Table 2. Overall,

\footnotetext{
${ }_{1}$ Assuming there is no recent flow cytometry to guide your decision on a patient. Each neurologist could only select one answer per age group.
}

high-efficacy agents were avoided and lower efficacy agents were preferred. Just $17 \%$ of neurologists reported they would still consider prescribing any of the DMTs in the context of COVID-19. The most commonly avoided agents in the context of COVID-19 were alemtuzumab (61\%), cladribine $(47 \%)$, ocrelizumab $(38 \%)$, rituximab $(35 \%)$, and natalizumab (34\%). Four percent of neurologists, however, reported they would not start a DMT at all in the context of COVID-19.

Questions specific to older patients in MS included a combination of open-ended and targeted questions. Impression on the number of weeks of an acceptable delay in dosing of a B-cell therapy for MS (i.e. rituximab or ocrelizumab) in patients 60 years and older versus $18-59$ years old are provided in Fig. 2 . $^{1}$

\section{Impressions of DMTs and future Covid19 situations}

Respondents were queried on whether certain DMTs "may not allow for a protective response to a potential SARSCoV-2 vaccine" with $80 \%$ agreeing with this statement and $20 \%$ in disagreement. Among those who thought DMTs may 


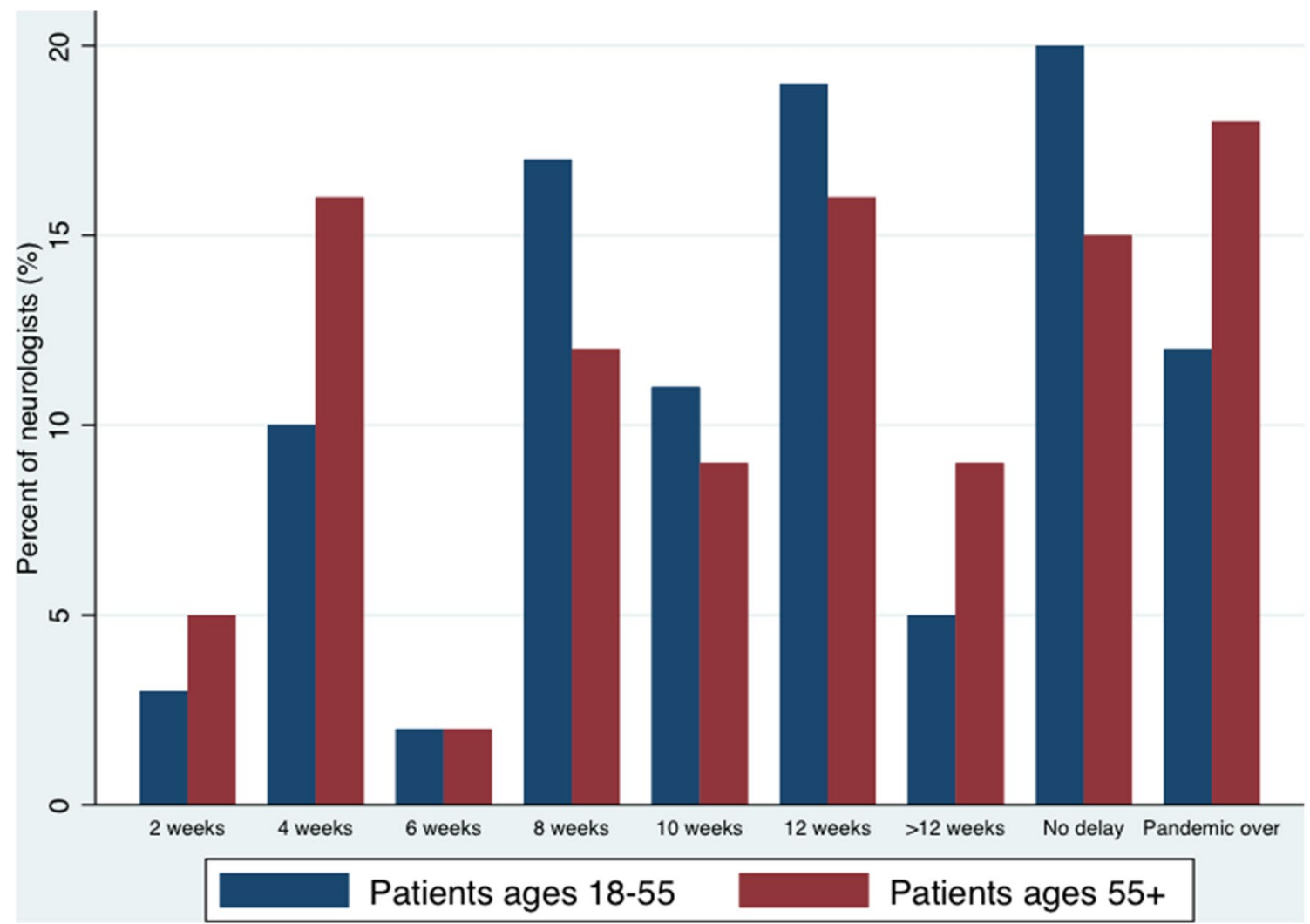

Fig. 2 Histogram of neurologists' $(n=243)$ response to:"For how long is it reasonable to delay the next B-cell therapy dose for your patients with MS during the COVID-19 pandemic?"
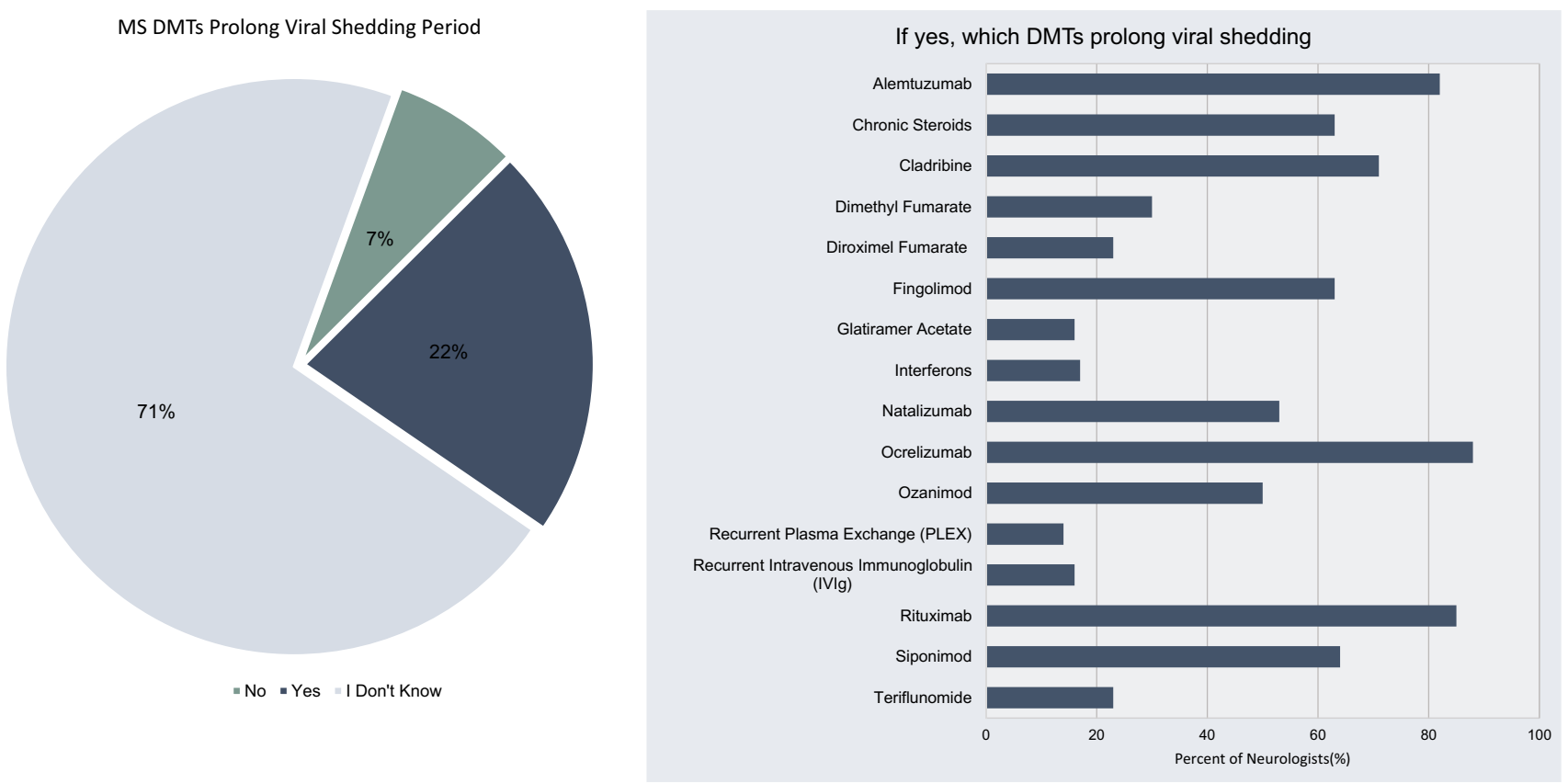

Fig. 3 Pie chart and bar graph of neurologists' $(n=243)$ response to: "Do you believe certain MS DMTs may prolong the period over which an MS patient sheds the virus? If so, which DMTs?" 
prohibit a protective vaccine response, the medications of highest concern were ocrelizumab (84\%), rituximab (83\%), alemtuzumab (78\%), cladribine (60\%), and chronic steroids (53\%) with all other DMT options leading to $<50 \%$ concern. Only $22 \%$ of neurologists believed that certain DMTs would prolong the period over which an MS patient sheds the novel coronavirus if exposed, whereas $71 \%$ stated they did not know. Among those who believed that a DMT could prolong viral shedding of the virus, ocrelizumab (87\% of respondents), ritxuximab (85\%), and alemtuzumab $(81 \%)$ were considered to be the most likely to do so (Fig. 3).

\section{Summary of qualitative responses}

Respondents were asked for their impressions of the COVID-19 situation for their patients and practices in open answer form with a focus on unmet needs for MS patients. A synthesis of the responses included a range of issues. One respondent stated: "This is the most difficult time I have seen in my 19 years of neurology." General needs included access to a vaccine and antiviral therapy for Sars-CoV-2. However, specific needs were prominent to MS patients including a focus on patients in rural areas, those without access to technology, older patients, patients who live alone, patients with advanced disability, patients with comorbid systemic illnesses, patients who could not access routine services due to their designated "elective" nature such as laboratory testing and infusions, access to coronavirus testing including acutely and for a future antibody, and access to psychological support for patients. A recurring need for more data on dosing, safety, reported COVID-19-MS cases, guidance on patients' return to work, and future vaccine efficacy were called for. Practice related issues included insurance barriers to home infusions, equity issues among health care workers (such that nurses and medical assistants were redeployed), financial strains, and reimbursements for telemedicine were noted. The was a general concern about the need to see some patients in person and the risk of future relapses in MS patients who were altering their current disease surveillance and treatment approaches.

\section{Discussion}

COVID-19 has led to several practice-changing situations and prescribing choices for neurologists as they approach the treatment of MS patients. Nearly all MS patients treated by responding neurologists were prescribed one of the nearly twenty available DMTs for MS prior to COVID-19. Most surveyed specialists believe that MS patients have a moderate to significantly increased risk of acquiring SARSCoV-2 (COVID-19) and believe certain DMTs are safer than others for MS patients' safety during the pandemic. Most neurologists have already made prescribing changes in the setting of COVID-19. At the time of this study, when data on safety of the various DMTs were lacking in MS patients, the opinion of experts drives decision-making, consensus guidelines, and patient outcomes. The USA accounted for $>1.5 \mathrm{M}$ cases of COVID-19 and >90,000 deaths, accounting for a third of the global cases and a quarter of the global deaths. Even at the time of publication, the USA continues to have one of the highest global rates of new COVID-19 infections. Since eradication of the novel coronavirus appears unlikely, particularly in the USA, the impact of community spread of the novel coronavirus may impact prescribing choices in MS for the foreseeable future.

An ongoing debate continues in the MS field on induction therapy or early use of higher efficacy agents versus escalation therapy which includes initiation of lower efficacy agents. Treatment "sequencing" may occur since most MS patients are likely to take multiple DMTs over the course of their lifetimes. The primary driving influence of this selection has often been efficacy of the DMTs with a focus on the incidence of MS relapses, new T2/FLAIR hyperintense lesions on MRI, and accrual of disability [14]. Additional considerations of safety, tolerability, and convenience have been included in this decision-making process. The COVID19 era has led to a potentially new balance in the expectations of patients and their prescribers as well as the impact on DMT selection, dosing, and continuance.

MS specialists differ in their approach to mitigating the risks of immunosuppression. Most (65\%) neurologists are doing at least one of: deferring DMT doses, changing the dose, changing the dosing interval, discontinuing DMTs altogether, switching to a different DMT, and for infused products in particular, changing to home infusions. Although there is also a strong tendency to make no changes, this occurs in only a minority of specialists. In the case of B-cell therapies, a similar number of neurologists responded that they would hold the medication until after the pandemic as would choose not to defer the next dose at all. Whether dosing and prescribing patterns will remain similarly disparate in the long term is uncertain. If more patients will remain off of DMTs permanently or "de-escalate therapy" to choose lower efficacy injectable and oral agents in higher proportions is uncertain. The longer term impact of the fear of COVID-19 may influence future new patient prescribing as well as in older MS patients (i.e. approximately above 55 years), the latter whom have the least available evidence for efficacy and are at higher risk of COVID-19.

The impact on DMTs on future COVID-19 vaccinations, if they are developed successfully, remains to be understood, particularly for DMTs with long-term effects, such as B-cell depleting therapies. Further studies on the dosing of DMTs as well as opportunities for extended dosing intervals and lower doses are needed for the higher efficacy agents. The 
possibility that DMTs may prolong viral shedding of the novel coronavirus is considered possible by many neurologists. Other unmet needs for scientific results include an understanding of whether any of the DMTs exert antiviral effects that are relevant to COVID-19 in a clinically meaningful way.

Approximately, a quarter of neurologists are aware of their MS patients self-discontinuing DMTs. MS specialists are aware of discontinuation in more than one in every 14 patients. Since physicians tend to over-estimate adherence in patients, and MS DMT adherence ranges from 46 to $97 \%$ in non-pandemic times [15-17], the number of people living with MS taking their DMTs on schedule is likely even lower than estimated here.

This report is unique in that it provides systemically collected data of a large group of MS-focused neurologists at a time when the novel 2019 SARS coronavirus was increasing in incidence across North America, when testing was variably present, and data on people with immunosuppression for MS as a subgroup with COVID-19 are not yet available. We queried a high number of respondents in a short period of time, allowing a snapshot in time of a moment in crisis in the country. However, the U.S. epidemic unfolded, and continues to unfold, in different ways in different regions and groups in the country, and differently in parts of Canada, reflecting the wave of COVID-19's impact on patient populations by both biological and non-biological variables. Some authors have suggested that immunosuppression is protective [18], although this remains speculative. Current clinical trials for the treatment of COVID-19 include several of the MS DMTs including the interferons, sphingosine 1-phosphate inhibitors, fumarates, teriflunomide, and natalizumab [19]. Equipoise exists in each of these trials. High throughput screening of available drugs has implicated interferons as potentially therapeutic for COVID-19 [20] given their presumed antiviral effects, a drug class now being tested in a global COVID-19 trial. In the 2 months since this study occurred, the general worry level among neurologists may have decreased. This may be due to reports out of Europe that show fewer cases than may have been expected [21], prognosis that is variable, and reduced infection rates. Strong mitigation strategies including strict social distancing, isolation, and avoidance of risk may have contributed to these numbers and needs to be further understood among MS patients. Guidelines now generally recommend continuation of MS DMTs with careful discussion around individual patients who take higher efficacy agents or have highest risk profiles [22, 23].

Our work is subject to multiple limitations, most notably relating to the sampling of neurologists. This is a sample of convenience. There is no one registry of expert physicians in neuroimmunology in the U.S.A. by which to know if our sampling is representative of the subspecialty density in the country. Our criteria included a minimum number of patients with MS seen in a 6-month period; however, we cannot rule out that respondents altered their responses in some way to improve their chance of inclusion or reported erroneously. Since this was introduced as an academic research study, no expectations of prescribing in a certain way or a certain drug would have been pre-suggested by our survey invitation. Respondents may have had differing views based on their location or time of response within the USA given that the incidence of cases and the surge of new cases were different, even in this 2.5 week period of time.

This survey was designed at the beginning of the pandemic in the U.S.A.; shortly afterwards, the impact of health disparities was even more apparent, including the differential impact of COVID-19 on lower income patient populations of African American and LatinX ancestry in the USA due to a variety of factors that are not race-related but socioeconomic. Our survey did not query the important specific issues on health disparities, access to telemedicine, or insurance status. Future surveys should consider these key variables more closely. Subgroup analyses were not performed in our dataset but could be performed in response to specific hypotheses in future work. The number of Canadian respondents made comparisons to the U.S. potentially under-powered. Similarly, studies of specific regions of the USA were not undertaken, since our unit of geographical classification was the state. Even within states (e.g. New York), different counties, cities, neighborhoods, and health facilities had differing COVID-19 experiences.

We are not aware of similar published academic research on this topic at the time of our study, making our results foundational for future other investigations on more targeted prescribing issues as they arise. In the time of social distancing, the risk of patients on immunosuppression is likely mitigated by structural changes in society. However, as various geographic regions "open up" and "normal routines" including work and eventually travel resume, added risk may ensue for community-acquired spread of coronavius to immunosuppressed MS patients. Large surveys performed rapidly across both academic and private practice settings are valuable to understanding the collective landscape in which MS patients and physicians navigate. Neuroimmunologists also have high financial value, with an estimated downstream revenue generation of 8 million USD for one full time equivalent and 25 million dollars for accompanying testing and prescribing. Although we did not study the financial impact of COVID-19 on neuroimmunology, our results imply that the financial costs to multiple parties will be high [24].

Taken together, COVID-19 has substantially disrupted usual patterns MS DMT practices. We provide a brief snapshot on the prevailing knowledge, attitudes, and practices of MS subspecialists during the COVID-19 pandemic. It 
is uncertain if these practice changes are temporary or will instead have lasting impact. The long-term consequences of the worry, perceptions, health behaviors, prescribing, and de-prescribing for MS patients remain to be measured.

\section{Availability of data and material}

Data from this study will be made available to qualified investigators upon request of the authors.

Acknowledgements We are grateful to our physician respondents for their time in completing the survey and ZoomRx for donating their survey platform support to the study.

Funding This study was supported by an unrestricted investigatorinitiated grant from Biogen, Inc.

\section{Compliance with ethical standards}

Conflicts of interest Mr. Alakel, Ms. Gazdag, and Mr. Kumar work for ZoomRx. All other authors declare there are no conflicts of interest.

Ethical standard statement The study was approved prior to commencement by the Partners Healthcare Institutional Review Board.

\section{References}

1. Wallin MT, Culpepper WJ, Campbell JD et al (2019) The prevalence of MS in the United States: a population-based estimate using health data. Neurology 92(10):e1029-e1040

2. Statistics Canada. Table 13-10-0467-01 Neurological conditions in household population. https://doi.org/10.25318/1310046701 -eng. Accessed 7 May 2020

3. No authors listed. National MS Society. Treating MS. https:// www.nationalmssociety.org/Treating-MS/Medications. Accessed 2 May 2020

4. Luna G, Alping P, Burman J et al (2019) Infection risks among patients with multiple sclerosis treated with fingolimod, natalizumab, rituximab, and injectable therapies. JAMA Neurol. https ://doi.org/10.1001/jamaneurol.2019.3365 (Epub ahead of print)

5. Willis MD, Robertson NP (2020) Multiple sclerosis and the risk of infection: considerations in the threat of the novel coronavirus, COVID-19/SARS-CoV-2. J Neurol 267(5):1567-1569

6. Holshue $\mathrm{ML}$, DeBolt C, Lindquist $\mathrm{S}$, for the Washington State 2019-nCoV Investigation Team et al (2020) First case of 2019 novel Coronavirus in the United States. N Engl J Med 382:929-936

7. Fuller T, Baker M (2020) Coronavirus death in California came weeks before first known U.S. death. NY Times. https://www. nytimes.com/2020/04/22/us/coronavirus-first-united-states-death .html.. Accessed 6 May 2020
8. Johns Hopkins University. Coronavirus Resource Center. https:// coronavirus.jhu.edu/us-map. Accessed 19 May 2020

9. Waldman G, Mayeux R, Claassen J et al (2020) Preparing a neurology department for SARS-CoV-2 (COVID-19): early experiences at Columbia University Irving Medical Center and the New York Presbyterian Hospital in New York City. Neurology 94(20):886-891

10. Brownlee W, Bourdette D, Broadley S, Killestein J, Ciccarelli O (2020) Treating multiple sclerosis and neuromyelitis optica spectrum disorder during the COVID-19 pandemic. Neurology 94(22):949-952

11. Centers for Disease Control and Prevention. Coronavirus (Covid19). https://www.cdc.gov/coronavirus/2019-ncov/index.html Accessed 26 April 2020

12. Multiple Sclerosis International Federation. https://www.msif. org/news/2020/02/10/the-coronavirus-and-ms-what-you-needto-know/ Accessed 9 April 2020

13. Bhatia R, Padma Srivastava MVP, Khurana D et al (2020) Consensus statement on immune modulation in multiple sclerosis and related disorders during the COVID-19 pandemic: expert group on behalf of the Indian Academy of Neurology. Ann Indian Acad Neurol 23(Suppl 1):S5-S14

14. Brown JWL, Coles A, Horakova D et al (2019) Association of initial disease-modifying therapy with later conversion to secondary progressive multiple sclerosis. JAMA 321(2):175-187

15. Menzin J, Caon C, Nichols C, White LA, Friedman M, Pill MW (2013) Narrative review of the literature on adherence to diseasemodifying therapies among patients with multiple sclerosis. J Manag Care Pharm 19(1 Suppl A):S24-40

16. Schreiber K, Kant M, Pfleger C, Jensen HB, Oesterberg O, Haid AR, Nielsen FK, Rubak S (2018) High treatment adherence, satisfaction, motivation, and health-related quality of life with fingolimod in patients with relapsing-remitting multiple sclerosis-results from a 24-month, multicenter, open-label Danish study. Patient Prefer Adherence 12:1139-1150

17. Erbay O, Usta Yeibalkan O, Yuceyar N (2018) Factors affecting the adherence to disease-modifying therapy in patients with multiple sclerosis. J Neurosci Nurs 50(5):291-297

18. Novi G, Mikulska M, Briano F, Toscanini F, Tazza F, Uccelli A, Inglese M (2020) COVID-19 in a MS patient treated with ocrelizumab: does immunosuppression have a protective role? Mult Scler Relat Disord 42:102120

19. Clinicaltrials.gov. Accessed 5 May 2020

20. Gordon DE, Jang GM, Bouhaddou M et al (2020) A SARS-CoV-2 protein interaction map reveals targets for drug repurposing. Nature. https://doi.org/10.1038/s41586-020-2286-9

21. Sormani MP (2020) An Italian programme for COVID-19 infection in multiple sclerosis. Lancet Neurol 19(6):481-482

22. MS treatment guidelines during coronavirus. National MS Society. https://www.nationalmssociety.org/coronavirus-covid-19-infor mation/multiple-sclerosis-and-coronavirus/ms-treatment-guide lines-during-coronavirus. Accessed 27 June 2020

23. Giovannoni G, Hawkes C, Lechner-Scott J, Levy M, Waubant E, Gold J (2020) The COVID-19 pandemic and the use of MS disease-modifying therapies. Mult Scler Relat Disord 39:102073

24. Berger JR (2017) The financial contribution of the multiple sclerosis specialist. Neurol Clin Pract 7(3):246-255 